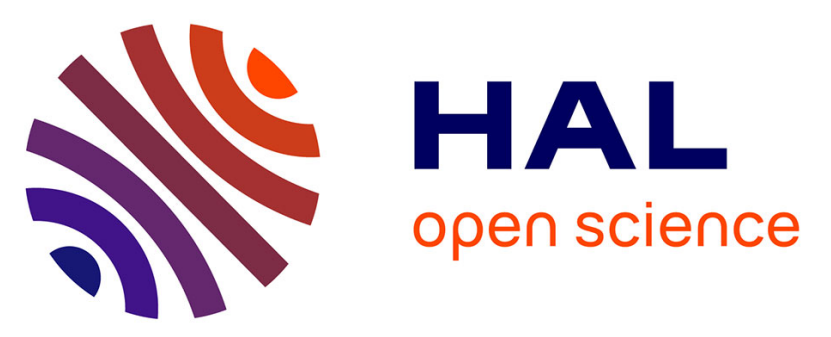

\title{
Contribution à l'étude des conditions optimales de la concentration optique dans les projets de centrales solaires. - Réduction de la tache de rayonnement fournie par un champ d'héliostats, au moyen de miroirs coniques optimisés.
}

P. Malifaud

\section{To cite this version:}

P. Malifaud. Contribution à l'étude des conditions optimales de la concentration optique dans les projets de centrales solaires. - Réduction de la tache de rayonnement fournie par un champ d'héliostats, au moyen de miroirs coniques optimisés.. Revue de Physique Appliquée, 1979, 14 (1), pp.21-33.

10.1051/rphysap:0197900140102100 . jpa-00244579

HAL Id: jpa-00244579

https://hal.science/jpa-00244579

Submitted on 1 Jan 1979

HAL is a multi-disciplinary open access archive for the deposit and dissemination of scientific research documents, whether they are published or not. The documents may come from teaching and research institutions in France or abroad, or from public or private research centers.
L'archive ouverte pluridisciplinaire HAL, est destinée au dépôt et à la diffusion de documents scientifiques de niveau recherche, publiés ou non, émanant des établissements d'enseignement et de recherche français ou étrangers, des laboratoires publics ou privés. 


\title{
Contribution à l'étude des conditions optimales de la concentration optique dans les projets de centrales solaires. Réduction de la tache de rayonnement fournie par un champ d'héliostats, au moyen de miroirs coniques optimisés.
}

\author{
P. Malifaud \\ Université Paris VI \\ Laboratoire, 95, Boulevard Jourdan, 75014 Paris, France \\ (Reçu le 12 juillet 1978, révisé le 6 novembre 1978, accepté le 8 novembre 1978)
}

\begin{abstract}
Résumé. - L'article rappelle la limite imposée à la concentration optique par le second principe de la thermodynamique et la relation de Clausius, et avance que les optiques instrumentales propres à approcher au mieux et le plus aisément cette limite sont des miroirs coniques ou pyramidaux particuliers. Une application à un projet de centrale solaire à champ d'héliostats est examinée en vue de projets ultérieurs. Des données détaillées sont exposées concernant le calcul pondéré des flux élémentaires cheminant dans un miroir conique et l'évaluation des pertes subies du fait des réflexions internes. Des informations technologiques sur les matériaux propres à assurer un grand coefficient de réflexion à des températures élevées et dans des conditions pratiques d'utilisation de longue durée à l'air libre complètent cette contribution.
\end{abstract}

\begin{abstract}
This paper narrates the limit imposed to the optical concentration by the second principle of thermodynamism and Clausius relation; and it outlines that the best optical instruments to attain this limit are particulars conical or pyramidal mirrors. An application for a solar plant with heliostats field is being examined, in view of other purpose. The datum are given in what concerns the balanced calculation of elementary flux through a conical mirror and the correct estimate of the losses coming from the inner reflections. Technological informations about proper materials giving a great reflective coefficient with high temperatures and in easy and lasting conditions at the open air, may complete this contribution.
\end{abstract}

1. Introduction. - L'auteur a développé dans sa thèse de doctorat [1] l'idée que l'étude de la concentration optique des rayonnements, sans formation d'image, avait intérêt à s'affranchir des conditions restrictives imposées traditionnellement aux systèmes à image (par lentilles, miroirs, sphériques, ou paraboliques, ...), conditions qui conduisent à des limitations d'ouverture relative et à des aberrations dispersives, maintenant ces systèmes bien en deçà des possibilités maximales de concentration. La concentration est ici entendue comme l'amplification d'un éclairement, définie par le rapport entre l'éclairement obtenu au moyen du dispositif concentrateur et l'éclairement direct.

Rappelons que la plupart des systèmes à image ont une ouverture relative ne dépassant pas $F: 1$, ce qui fournit, dans l'air, une concentration 4 fois moindre que la concentration limite (et $4 n^{2}$ fois s'il y a immersion). Et si l'on donne à un miroir parabolique, par exemple, l'ouverture maximale, jusqu'au plan de son foyer, l'éclairement maximal ne serait atteint, théoriquement, dans l'image dite de gauss (de diamètre $=f . \alpha$ ) qu'au prix d'une perte de flux de $75 \%$. Le rapport du flux arrivant dans l'image de gauss au flux capté est en effet égal à $\cos ^{4} \theta / 2$ (formule de Maréchal et Slansky), soit ici 0,25 . En outre, l'expérience montre que dans les meilleurs cas l'éclairement moyen réel dans l'image de Gauss ne dépasse pas le tiers de l'éclairement limite calculé.

Au contraire, il a été établi [1] qu'un miroir conique optimisé peut, dans les mêmes conditions, approcher effectivement l'éclairement limite avec de faibles pertes de flux.

1. La concentration maximale. Les miroirs coniques optimisés. - Ce n'est pas ici le lieu de démontrer et d'étayer expérimentalement cette propriété des miroirs coniques. Un article en cours pour la Revue d'Optique fera le point sur la question. Rappelons simplement qu'un théorème de la concentration maximale a été 
établi, qui peut se résumer ainsi : Etant donné une surface émettrice, un système optique (quel qu'il soit) et une surface réceptrice, pour que l'éclairement sur cette dernière soit maximal, il faut et il suffit que cette surface reçoive le rayonnement exactement comme elle l'émettrait si elle était un Corps Noir émetteur, c'est-à-dire selon la loi de Lambert. Il s'ensuit, pour un dispositif expérimental, que tout rayon construit géométriquement à partir de la surface réceptrice (et pas seulement par retour inverse, bien entendu) passe par la surface émettrice. Une étude approfondie montre que cette condition n'est réalisée qu'au prix d'une augmentation de l'étendue optique globale du faisceau capté (l'étendue élémentaire étant, comme on le sait, $n^{2} \mathrm{~d} s \cos \theta \mathrm{d} \omega$ ), autrement dit d'une perte de flux corrélative à la concentration. C'est ce qu'il a été proposé d'appeler l'entropie optique [2]. Cette perte théorique est de $75 \%$ pour les miroirs paraboliques, comme on l'a rappelé. Elle serait théoriquement nulle (cas limite) pour une fibre conique d'angle au sommet négligeable (et d'absorption et pertes supposées nulles). Mais les miroirs coniques intéressants dans la pratique sont ceux qui ont un angle au sommet aussi grand que possible, afin que soient limités leur longueur et le nombre des réflexions internes. Ce sont eux qui ont fait l'objet des optimisations proposées.

1.1 LeS DEUX SECTIONS OPTIMALES. Formules GÉNÉRALES. - Pour un miroir conique de demi-angle au sommet $\gamma$, captant un rayonnement sous un angle maximal d'incidence $\theta$ dans sa grande section droite d'entrée de flux de surface $s_{1}$, il existe deux sections optimales $s_{2}$ et $s_{3}$. La figure 1 résume ce résultat sur un exemple. Le flux capté se conserve intégralement (aux pertes par réflexion près, qui seront évaluées plus

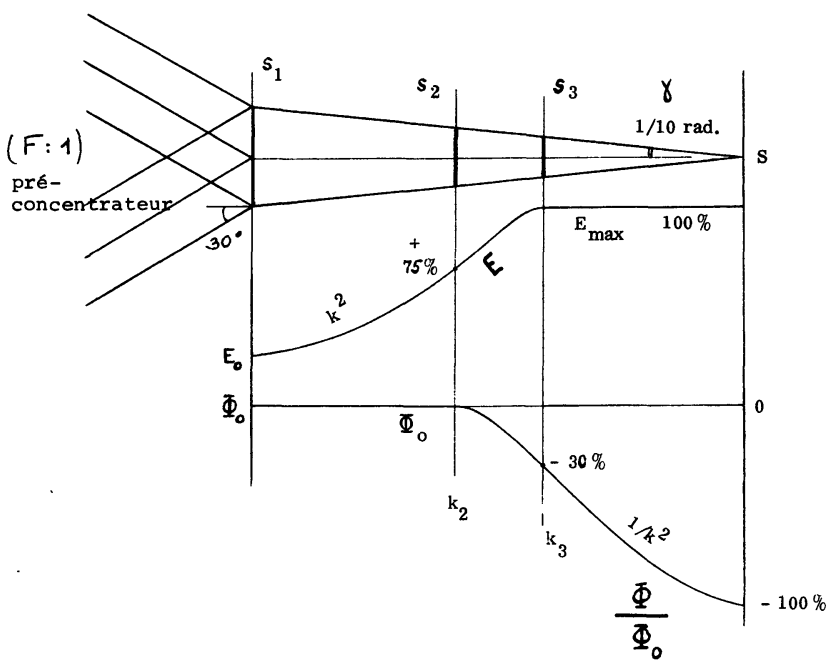

Fig. 1. - Variations de l'éclairement et du flux dans les sections d'un miroir conique. $k=$ rapport du $\varnothing$ de la section d'entrée au $\varnothing$ de la section considérée.

[Variations of the illuminance and of the flux in the sections of a conical mirror. $k=$ account of diameter of the inlet section to the diameter of the considered section.] loin) jusqu'à une certaine section $s_{2}$, donnée par la formule approchée :

$\frac{s_{1}}{s_{2}}=\frac{\cos ^{2} \gamma}{\sin ^{2}(\theta+\gamma)}$

tandis que l'éclairement croît jusqu'à atteindre en $s_{2}$ une valeur $E_{2}$ dont le rapport à l'éclairement maximal limite est :

$\frac{E_{2}}{E_{\max }}=\frac{1}{\left(1+\operatorname{tg} \gamma \sqrt{\left.\frac{1}{\sin ^{2} \theta}-1\right)^{2}}\right.}$

Cette première optimisation permet donc d'obtenir le plus grand éclairement possible sans consentir de perte de flux.

Au-delà de $s_{2}$, l'éclairement continue à croître jusqu'à atteindre la valeur maximale limite en une certaine section $s_{3}$, donnée par la formule approchée

$\frac{s_{1}}{s_{3}}=\frac{\cos ^{2} \gamma}{\sin ^{2}(\theta-\gamma)}$

tandis que le flux diminue en passant par une valeur $\Phi_{3}$ en $s_{3}$ et en tendant ensuite vers zéro lorsque la section réceptrice se rapproche du sommet du cône. La section $s_{3}$ caractérise donc une seconde optimisation permettant d'atteindre l'éclairement limite en consentant la plus petite perte de flux possible, laquelle est donnée par la formule approchée :

$\frac{\Phi_{3}}{\Phi_{0}}=\left(1-\operatorname{tg} \gamma \sqrt{\frac{1}{\sin ^{2} \theta}-1}\right)^{2}$

Dans les deux cas, il a été calculé que le nombre moyen pondéré, $\bar{p}$ de réflexions à prendre en compte, est, avec une bonne approximation :

$\bar{p}=0,64 \frac{\frac{\pi}{2}-\theta}{2 \gamma}$.

1.2 Applications. - Le système peut fonctionner dans les deux sens, soit comme concentrateur en guidant le rayonnement d'une grande section vers une petite, soit comme projecteur en associant une source à une petite section et en guidant le rayonnement vers une grande section. Sur ces bases, ont été étudiés et réalisés depuis une dizaine d'années des concentrateurs et des projecteurs de rayonnements, de toutes sortes. Cf. [3], [4], [5], [6], [7], [8], [9], [10], [11], [12], [13], [14].

Dans le cas de la concentration, le miroir conique est, en général, associé à une optique pré-concentratrice frontale, afin que l'angle $\theta$ (d'incidence maximale à l'entrée) ne soit pas trop petit, ce qui entraînerait une grande longueur pour le cône et un grand nombre de réflexions. Le miroir conique a 
pour fonction de parachever cette pré-concentration. Dans l'exemple de la figure 1, cette optique serait un simple condenseur d'ouverture classique $\mathrm{F}: 1$. L'étude expérimentale a confirmé les résultats prévus, ce, malgré la qualité médiocre de tels condenseurs.

2. Application à la concentration du rayonnement solaire. - Les applications à la concentration du rayonnement solaire posent des problèmes particuliers, du fait de la nature des optiques pré-concentratrices qui sont, soit des miroirs paraboliques, soit des champs d'héliostats convergents, et du fait des conditions thermodynamiques d'optimisation des chaudières ou des récepteurs associés, mettant en cause la distribution de l'énergie concentrée en fonction notamment, de l'angle d'émergence à la sortie du miroir conique.

Les applications visant à l'obtention de températures très élevées, au prix d'une perte de flux limitée au minimum, par une concentration approchant la limite (seconde optimisation) ont été déjà évoquées dans un article de 1971 [15]. C'est un type très important d'applications. D'autres publications lui seront consacrées. La première optimisation, visant à réduire le diamètre de la tache concentrée sans consentir de perte théorique du flux, fait plus spécialement l'objet du présent article.

2.1 OPTIMISATION SANS PERTE DE FLUX D'UN MIROIR PARABOLIQUE ET D'UN MIROIR CONIQUE. - Il faut d'abord rappeler que les formules d'optimisation sont tout à fait différentes des formules générales indiquées plus haut, lorsque l'optique frontale associée est de nature parabolique. La concentration moyenne dans l'image totale fournie par un miroir parabolique de demi-angle d'ouverture $\theta$, captant un rayonnement sous un angle apparent $\alpha$, est égale à $\frac{\sin ^{2} 2 \theta}{\alpha^{2}}$. La concentration propre au miroir conique associé étant, en première optimisation (1) : $\frac{\cos ^{2} \gamma}{\sin ^{2}(\theta+\gamma)}$, la concentration $C$ obtenue finalement par la combinaison est :

$C=\frac{1}{\alpha^{2}} \frac{\sin ^{2} 2 \theta}{(\sin \theta+\operatorname{tg} \gamma \cos \theta)^{2}}$.

Le rapport $\zeta$ à la concentration limite, dont une expression connue est $4 / \alpha^{2}$, est donné par la formule approchée :

$\zeta=\left(\frac{\sin \theta}{\operatorname{tg} \theta+\operatorname{tg} \gamma}\right)^{2}$

A $\gamma$ constant, la dérivée s'annule pour $\operatorname{tg} \gamma=\operatorname{tg}^{3} \theta$. A une valeur donnée de $\gamma$, une valeur donnée de $\theta$ correspond, pour laquelle la fonction de concentration présente un maximum, et l'on a :

$\zeta_{\max }=\cos ^{6} \theta$.
L'optimisation présente donc un degré de liberté de moins que dans le cas général. On a représenté sur la figure 2 les variations de la fonction (7), à $\gamma$ constant, et de la fonction (8).

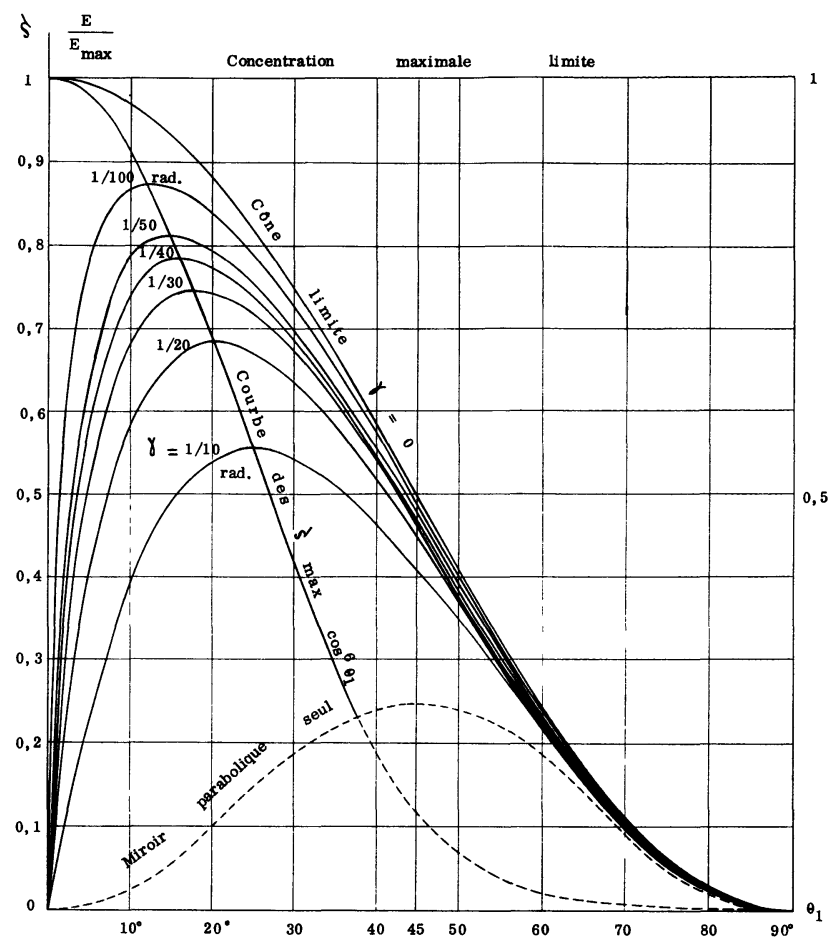

Fig. 2. - Courbe de $\zeta$ en fonction de $\theta_{1}$ à $\gamma$ constant. [Curves of $\zeta$ versus $\theta_{1}$ to $\gamma$ constant.]

Si on exprime la fonction $\zeta$ à $\bar{p}$ constant (nombre moyen pondéré de réflexions internes), on trouve :

$$
\zeta=\left(\frac{\sin \theta}{\operatorname{tg} \theta+\operatorname{tg} \frac{1-\frac{2}{\pi} \theta}{2 \bar{p}}}\right)^{2}
$$

dont la dérivée s'annule pour

$\bar{p}=\frac{\frac{\pi}{2}-\theta-\operatorname{tg} \theta}{2 \operatorname{tg}^{3} \theta}$

D'où l'on tire :

$\zeta_{\max }=\cos ^{6} \theta\left(1+\frac{2 \sin ^{3} \theta}{\cos \theta(\pi-2 \theta \sin 2 \theta)}\right)^{2}$.

Les courbes de la figure 3 représentent les variations de $\bar{p}$ pour des valeurs données de $\zeta$.

2.2 ETUde D'Une APPLICATION DANS LE CADRE DU PROJET THÉMIS. - Dans le projet de centrale solaire "THÉMIS », par exemple, mis en œuvre par le P.I.R.D.E.S. [16], un champ d'héliostats à courbure pseudo-parabolique fournit une tache concentrée 


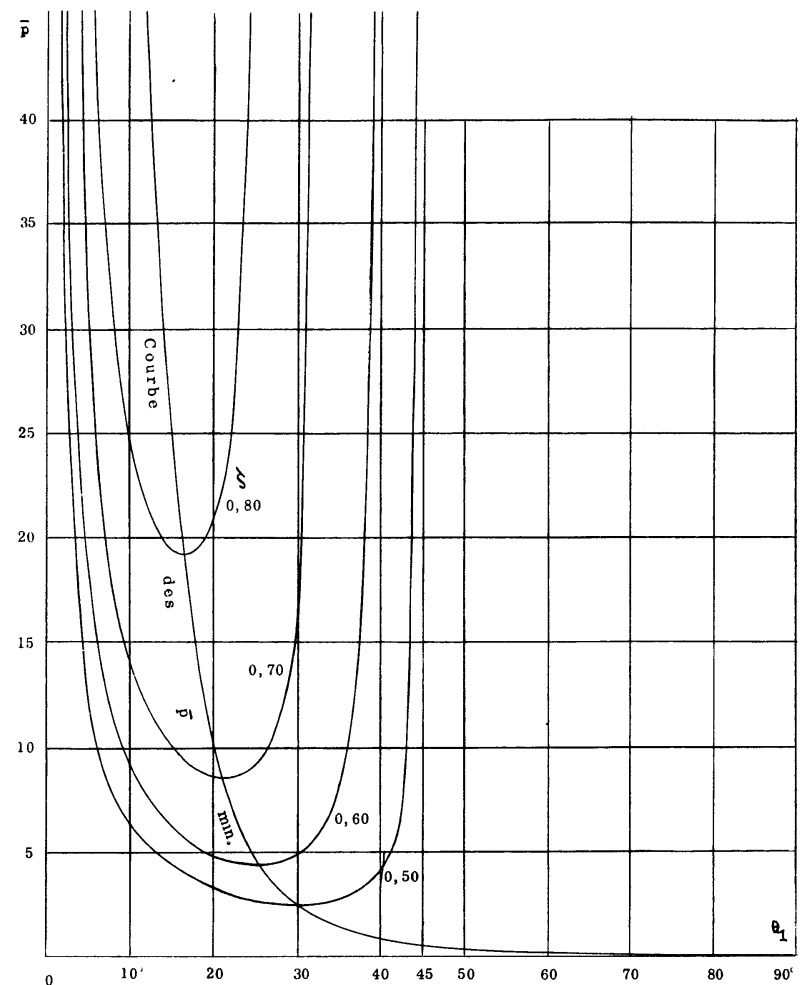

Fig. 3. - Courbes de $\bar{p}$ en fonction de $\theta_{1}$ pour $\zeta$ prédéterminé. [Curves of $\bar{p}$ versus $\zeta$ for $\theta_{1}$ predetermined.]

de 6,50 $\mathrm{m}$ environ de diamètre, reçue dans l'entrée d'une chaudière. La figure 4 montre une coupe schématique axiale verticale de l'ensemble. La figure 5 est le plan des 325 héliostats. La question s'est posée de savoir s'il y aurait intérêt à réduire le diamètre de cette tache sans perdre de flux (autrement que par réflexion), en augmentant en outre l'angle d'entrée du faisceau dans la chaudière et en élevant la concentration donc la température, dans cette entrée. Ce que peut faire un miroir conique enpremière optimisation.

Dans cet exemple, l'implantation du champ d'héliostats, judicieusement conçue pour une captation directe par la chaudière, antérieurement aux considérations exposées ici, s'est avérée peu favorable à l'adjonction d'un miroir conique associé. En effet, l'angle maximal $\theta$ des faisceaux fournis par les héliostats est très grand, ce qui ne permet pas de faire jouer efficacement son rôle au miroir conique. On voit par les formules (1) et (2) (ou encore sur la figure 2), qu'une valeur de $\theta$ approchant les $60^{\circ}$, comme c'est le cas, ne permet pas d'optimisation valable. Même en

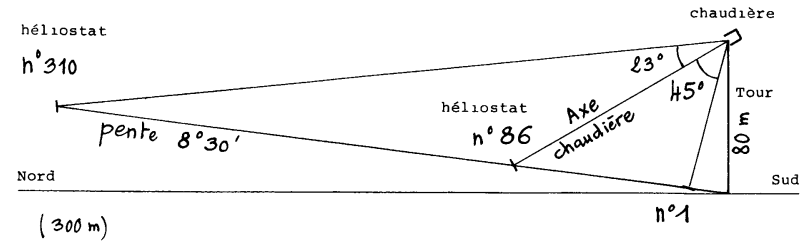

Fig. 4. - Coupe schématique axiale verticale de l'ensemble Thémis. [Axial and vertical schematic section of the Themis plant.]

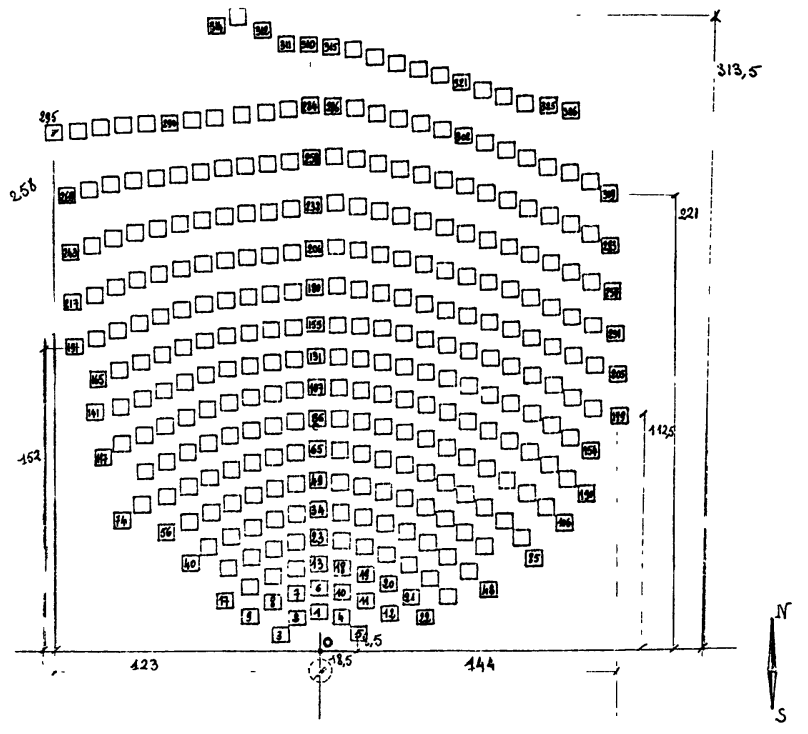

Fig. 5. - Plan de l'implantation des héliostats dans le projet de centrale Thémis.

[Plan of the implantation of heliostats in the project Themis.]

négligeant les héliostats extrêmes et en ramenant $\theta$ à une valeur un peu plus petite, on est bien loin $\mathrm{du}$ compte.

On peut se demander s'il faut ici se placer dans le cas général ou dans le cas de l'association avec une optique parabolique. Peu importe en effet, la forme du support des héliostats. S'ils se comportent comme des éléments paraboliques, c'est le second cas qu'il faut considérer. Mais si la répartition qu'ils fournissent au foyer est celle d'une optique grosso modo aplanétique (et non stigmatique), c'est le cas général qu'il faut considérer. Or, il semble que le comportement de ce champ d'héliostats s'apparente à celui d'une lentille de Fresnel. Si une ćtude est reprise dans des conditions nouvelles définies plus loin, il faudra élucider à quelle classe de répartition optique il est préférable de se rattacher et reconsidérer en conséquence la distribution et peut-être la conception même des héliostats.

Dans l'étude préliminaire qui a été faite, cette question revêtait peu d'importance car les deux modes de calcul donnaient des résultats voisins dans les conditions inadéquates où l'on se trouvait. Après une longue recherche de compromis, au demeurant peu satisfaisants, un miroir conique a été défini, susceptible d'améliorer quelque peu l'exploitation de la tache par la chaudière.

Ce miroir (Fig. 6 et 7) est pseudo-conique, la grande section d'entrée de flux étant un cercle de $6,50 \mathrm{~m}$ de diamètre, la petite section, une ellipse ayant un grand axe horizontal de $5,55 \mathrm{~m}$ et un petit axe de $4,60 \mathrm{~m}$; la longueur dans l'axe du miroir étant de $5 \mathrm{~m}$. Les axes des sections ne coïncident pas. L'axe de la petite section (pupille d'entrée de la chaudière) est parallèle à l'axe de la grande section, à $1 \mathrm{~m}$ au-dessus de celui-ci 


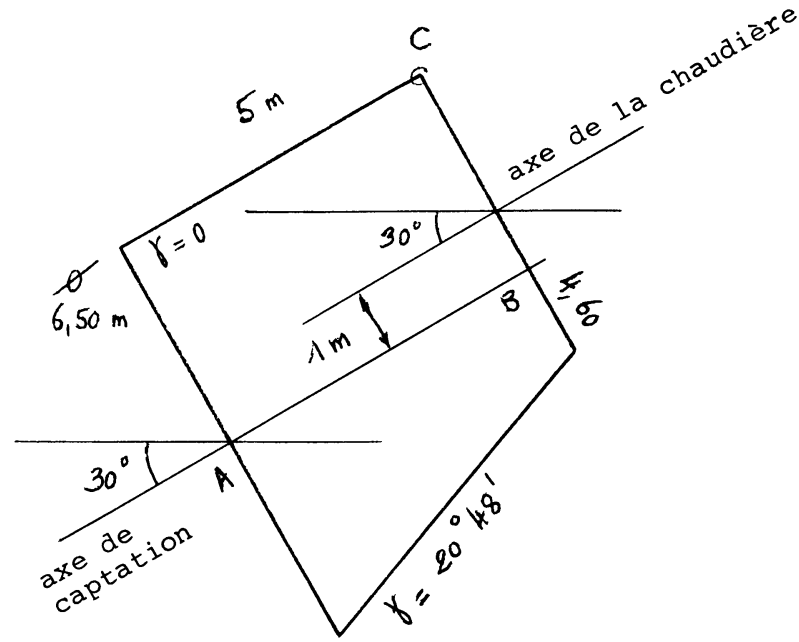

Fig. 6. - Coupe schématique axiale verticale du miroir tronconoïdal (étude pour Thémis).

[Axial vertical schematic section of a mirror (Themis).]

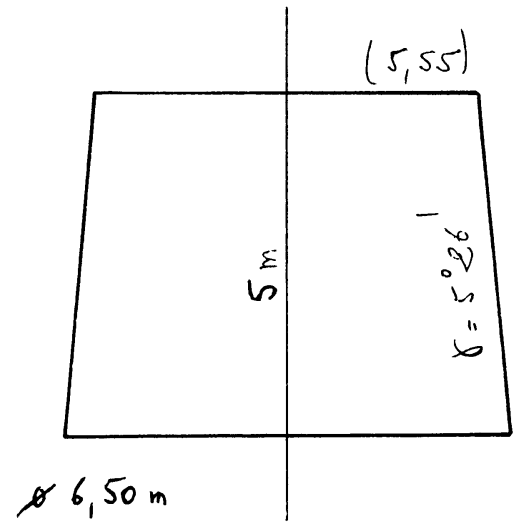

Fig. 7. - Coupe longitudinale transversale AB.

[Longitudinal and cross section.]

dans le plan vertical. Ce miroir serait constitué d'une centaine de trapèzes plans en feuilles d'aluminium A 99 traité par le procédé dit brytal, ayant chacun une grande base de l'ordre de $0,20 \mathrm{~m}$, une petite base de l'ordre de $0,15 \mathrm{~m}$, une longueur de l'ordre de $5,30 \mathrm{~m}$ à $5 \mathrm{~m}$. Ces trapèzes seraient fixés sur une armature externe délimitant les sections d'entrée et de sortie de flux.

Le plan de focalisation des héliostats serait avancé de $5 \mathrm{~m}$, de manière à coïncider avec la section d'entrée du miroir.

Les avantages à attendre d'un miroir conique, en général, sont de diminuer les pertes par convexion en réduisant l'entrée de la chaudière et de fournir à celle-ci un faisceau de rayonnement plus ouvert et plus homogène. Dans les conditions imposées ici au départ, l'obtention de ces avantages est aléatoire. La figure 8 donne les courbes de rendements établies théoriquement par un membre de l'équipe du projet THEM. La courbe A est celle du rendement optique en fonction du diamètre de la tache captée par la chaudière.

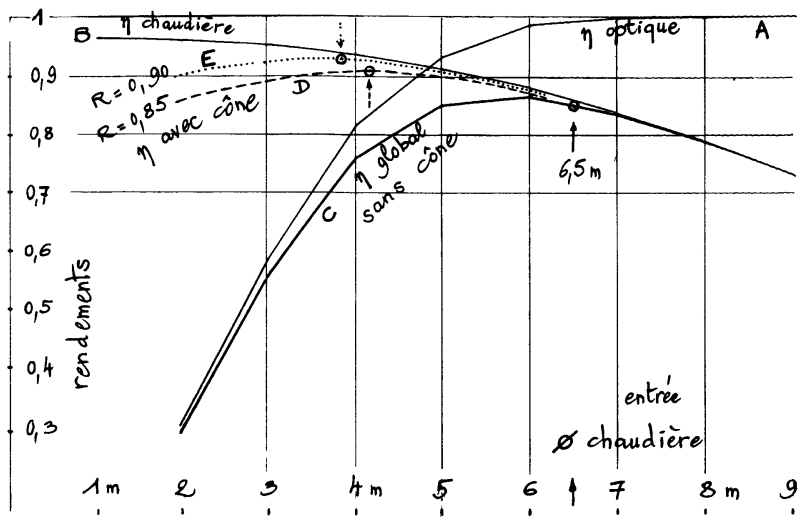

Fig. 8. - Courbes de rendements sans cône et avec cône (étude pour le projet Thémis).

[Curves of efficiency without cone and with cone.]

La courbe B est celle du rendement propre de la chaudière. La courbe $C$ est celle du rendement global résultant du produit des deux premiers. La courbe $\mathrm{D}$ est celle du rendement global attendu avec l'adjonction d'un miroir conique ayant un coefficient de réflexion de 0,85 . La courbe $\mathrm{E}$, pour un coefficient de réflexion de 0,90 . Le diamètre d'entrée de la chaudière étant supposé réduit au-dessous de $5 \mathrm{~m}$, il était entendu qu'aucun des rayons pris en charge par le miroir conique ne subirait plus d'une réflexion. On voit qu'on peut attendre théoriquement un gain de rendement de l'ordre de $5 \%$ dans le cas d'un coefficient de réflexion de 0,85 et un diamètre de l'ordre de 4 à $4,50 \mathrm{~m}$, et de l'ordre de $7 \%$ avec 0,90 et un diamètre de 3,5 à $4 \mathrm{~m}$. Mais ceci est aléatoire, ces courbes comportant des incertitudes difficiles à évaluer et le compromis adopté pour le miroir pseudo-conique n'entrant pas dans le cadre normal des optimisations des miroirs coniques. Autant dire que cette étude préliminaire ne débouche pas de manière évidente sur une application sûre à court terme pour le projet actuellement en cours de réalisation de la centrale THEMIS. Son intérêt est plutôt d'avoir clarifié une question difficile et d'avoir permis de préciser, en vue de projets ultérieurs les conditions optimales du recours à un miroir tronconique.

2.3 CONDITIONS OPTIMALES POUR UN PROJET ULTÉRIEUR. - La première condition sera de modifier l'implantation du champ des héliostats. Il faudrait que l'angle d'incidence dans le plan de la tache focale n'excède pas $30^{\circ}$ en moyenne et que la répartition angulaire soit davantage symétrique et même, si possible, prédéterminée. Ceci conduirait à adopter un site présentant une pente plus accentuée, l'idéal étant de fournir au miroir conique un cône de rayonnement de demi-angle au sommet d'au plus $30^{\circ}$. Il ressort de la théorie que l'efficacité d'un miroir conique est bien meilleure pour un angle d'incidence inférieur à environ $30^{\circ}$. Le champ d'héliostats fournirait alors une tache plus grande, mais le miroir 
conique la ramènerait à une dimension approchant celle de l'image de Gauss. Dans une réalisation similaire à celle de l'actuel projet THÉMIS, les 325 héliostats seraient, par exemple, répartis dans un triangle ayant son sommet à une cinquantaine de mètres de la tour, une base de l'ordre de $150 \mathrm{~m}$, à $350 \mathrm{~m}$ de la tour. Mais il vaudrait mieux repenser tout le problème.

Le miroir tronconique associé serait de révolution. Il serait commodément réalisé au moyen de feuilles planes d'aluminium A 99 (vendues polies), traitées, découpées selon la surface latérale développée du tronc du cône. Les miroirs coniques ne constituant pas des optiques à image, leur surface latérale peut présenter, sans inconvénient, de légères déformations et gauchissements et la tolérance des cotes est grande. Pour $\theta=30^{\circ}$, si son diamètre d'entrée était, par exemple, de l'ordre de $6,50 \mathrm{~m}$, son diamètre de sortie serait de l'ordre de $3 \mathrm{~m}$. Sa longueur, de l'ordre de 7 à $8 \mathrm{~m}$. La concentration à la sortie serait de l'ordre de 7000 soleils. Le nombre moyen pondéré de réflexions, $\bar{p}$, de 1,3 environ. L'angle à la sortie (angle d'incidence dans la chaudière) approcherait de $90^{\circ}$ et la répartition de l'énergie serait beaucoup plus régulière entre les directions d'incidence. De l'avis des spécialistes, ces nouvelles conditions présenteraient un réel intérêt. Pour $\theta=25^{\circ}$, on aurait un ordre de 10000 soleils pour un diamètre d'entrée de $2,50 \mathrm{~m}$. Pour $\theta=20^{\circ}, 15000$ soleils et un diamètre de $2 \mathrm{~m}$. Ces derniers chiffres sont donnés à titre indicatif. Les projets actuels de centrales à tour ne demandent pas des valeurs aussi élevées de concentration, mais on peut penser que des projets futurs faisant appel à des réacteurs chimiques ou à des cycles thermodynamiques à haute température, pourraient tirer parti des possibilités ainsi ouvertes. Par ailleurs, il ne serait pas nécessaire que les héliostats soient pseudo-paraboliques et fournissent une focalisation très performante, car ce serait le miroir conique qui parachèverait la concentration désirée. La courbure pourrait être sphérique ou pseudo-sphérique comme dans le cas des plaques de verre déformées selon des techniques très simples, par exemple celles proposées, naguère, par Monsieur Ivan Peychès. La focalisation serait sans doute moins bonne qu'avec des miroirs paraboliques ou des miroirs à courbure soignée, mais le prix de revient est intéressant. La tache finale fournie par le miroir tronconique serait ellemême légèrement plus grande, mais l'inconvénient serait minime eu égard à la grande concentration obtenue. Enfin, des miroirs en aluminium poli traité pourraient être envisagés.

Un autre point examiné dans l'étude préliminaire qui vient d'être évoquée, est celui de la sécurité. Qu'arriverait-il si, pour une raison quelconque un dépointage se produisait, envoyant la tache concentrée par les héliostats sur une paroi latérale du miroir conique ? Examinons la question dans la perspective d'une optimisation ultérieure dans de bonnes conditions, par exemple, à $\theta=30^{\circ}$. Le demi-angle au sommet du cône serait de l'ordre de $15^{\circ}$. L'éclairement énergétique serait multiplié par sin $15^{\circ}$, soit 0,26 . $\mathrm{Si}$ la concentration fournie par les héliostats était de 1500 soleils (chiffre plausible avec une combinaison de ce genre, dans l'exemple envisagé), la paroi d'aluminium encaisserait $1500 \times 0,26=390$ soleils. Le facteur d'émissivité calorifique de l'aluminium poli traité, à l'air libre, à 400, est inférieur à 0,05 (cf. [17]). On arrive à une concentration efficace de moins de 20 , ce qui correspondrait à moins de $700 \mathrm{~K}$, soit moins de $427^{\circ} \mathrm{C}$ (bien moins encore pour $\theta=25^{\circ}$ et $\theta=20^{\circ}$ ). Ce chiffre est calculé sans tenir compte d'aucun refroidissement. Le seul fait d'être à l'air libre le ramènerait au-dessous de $300^{\circ} \mathrm{C}$. Or, l'aluminium fond à $660^{\circ} \mathrm{C}$ et le traitement brytal ne subit pas de modification au-dessous de $550^{\circ} \mathrm{C}$ (voir la dernière partie de cet article). La déviation de l'axe du faisceau serait de $30^{\circ}$. La question se posera de savoir si la chaudière peut s'accommoder d'une telle déviation. Dans le cas, journalier, des ajustements de pointage matinaux, l'énergie supportée serait beaucoup plus faible.

Bien entendu, il sera toujours possible, en cas de besoin, de prévoir un refroidissement par eau, notamment au voisinage de la petite section du miroir tronconique.

3. Eléments de calcul des flux mis en auvre. Les formules approchées définissant les optimisations et le nombre moyen de réflexions internes ont été données dans le cas général d'un système pré-concentrateur aplanétique ou assimilé et dans le cas particulier d'un système pré-concentrateur parabolique. Un point important pour établir un projet de centrale solaire en faisant un choix des paramètres qui tienne compte des conditions optimales de rendement de la chaudière réceptrice, est de connaître la pondération énergétique exacte de chacun des pinceaux élémentaires captés et transformés par le miroir tronconique. En vue d'éventuels projets futurs, les données de base permettant de faire un calcul pondéré des flux élémentaires parvenant en toute section, et les résultats sont ici exposés. Un développement complet, se trouve dans la thèse [1], pages 60 à 275 .

3.1 Conduite DU CAlCul. - La théorie des miroirs coniques a d'abord été faite en considérant les rayons méridiens (dans un plan contenant l'axe du cône) puis en montrant que tout rayon non méridien peut être assimilé à un rayon méridien qui cheminerait dans un cône fictif appelé cône équivalent pour ce rayon non méridien.

La figure 9 montre la marche d'un rayon méridien; la figure 10, la marche d'un rayon non méridien. Les figures 11 et 12 font comprendre ce qu'est le cône équivalent pour ce rayon non méridien. C'est un cône fictif, composite (Fig. 11), de demi-angle au sommet $\alpha$.

Il suffit, pour disposer des éléments de calcul des flux, de partir de la relation entre les paramètres caracté- 


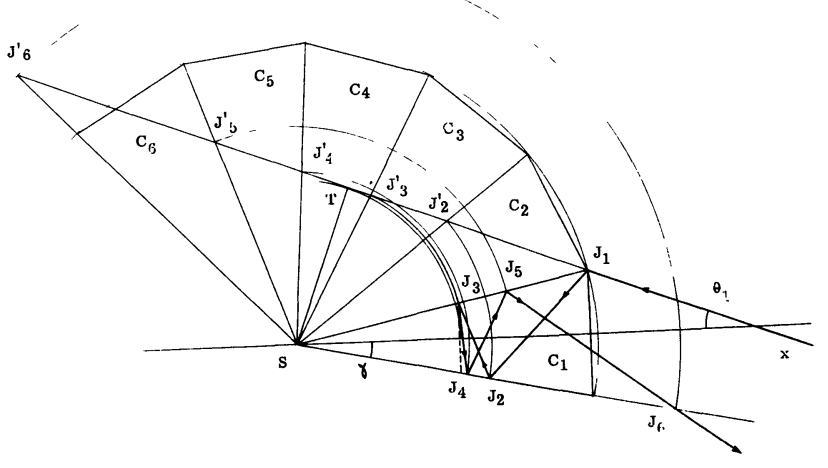

Fig. 9. - Cheminement d'un rayon méridien dans un miroir conique.

[Carrying out of a meridian ray in a conical mirror.]

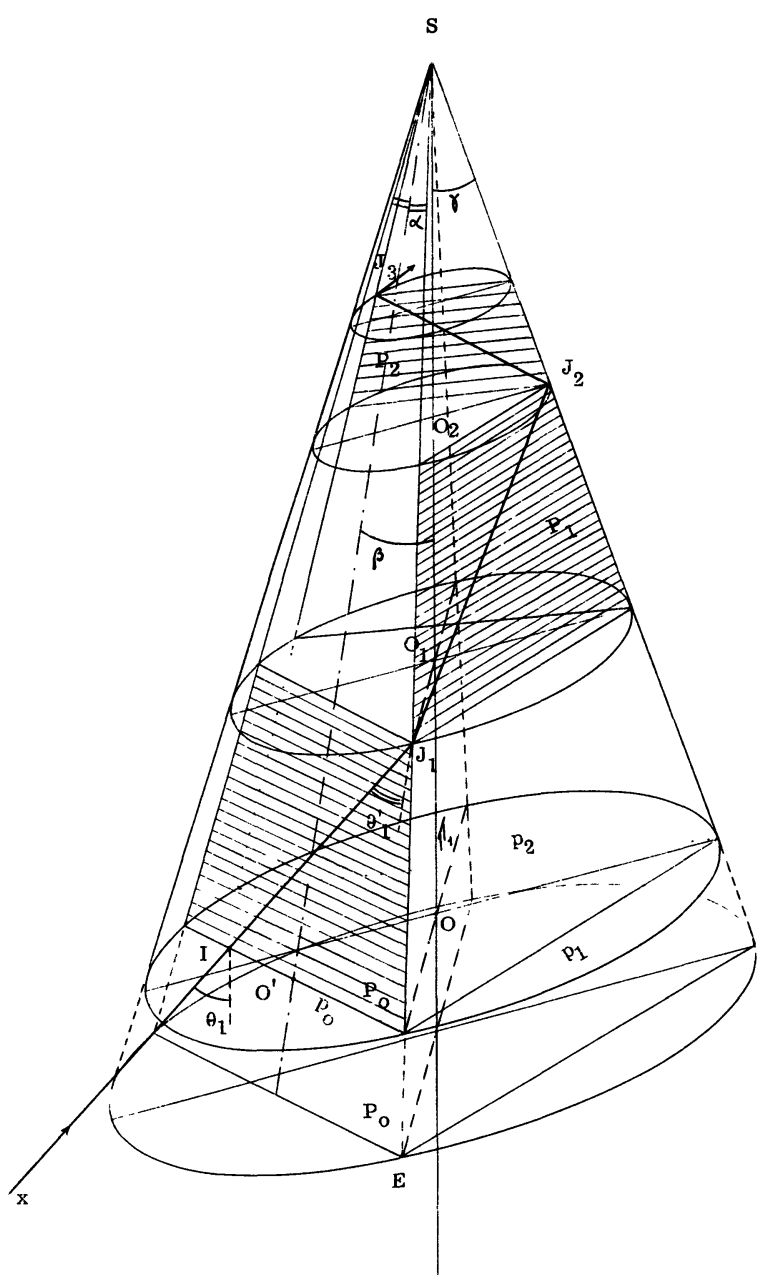

Fig. 10. - Cheminement d'un rayon non méridien dans un miroir conique.

[Advancing of a non-meridian ray in a conical mirror.]

risant tout rayon incident et le demi-angle au sommet $\alpha$ du cône équivalent pour ce rayon.

3.1.1 Pondération du flux à l'entrée du miroir conique. Hypothèses du calcul. — Un rayon $x I$ incident

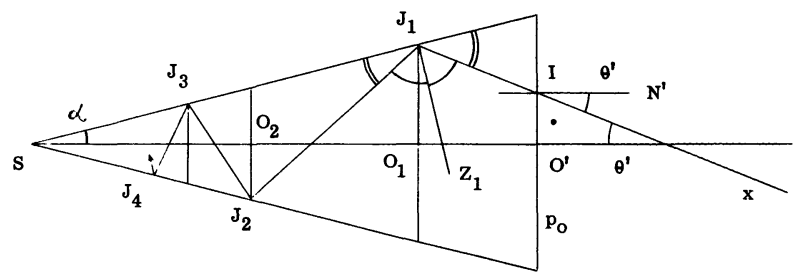

Fig. 11. - Cône équivalent pour un rayon non méridien. Coupe composite reconstituée dans un plan.

[Similar cone for a non-meridian ray. Multiple section reconstitued in a plan.]

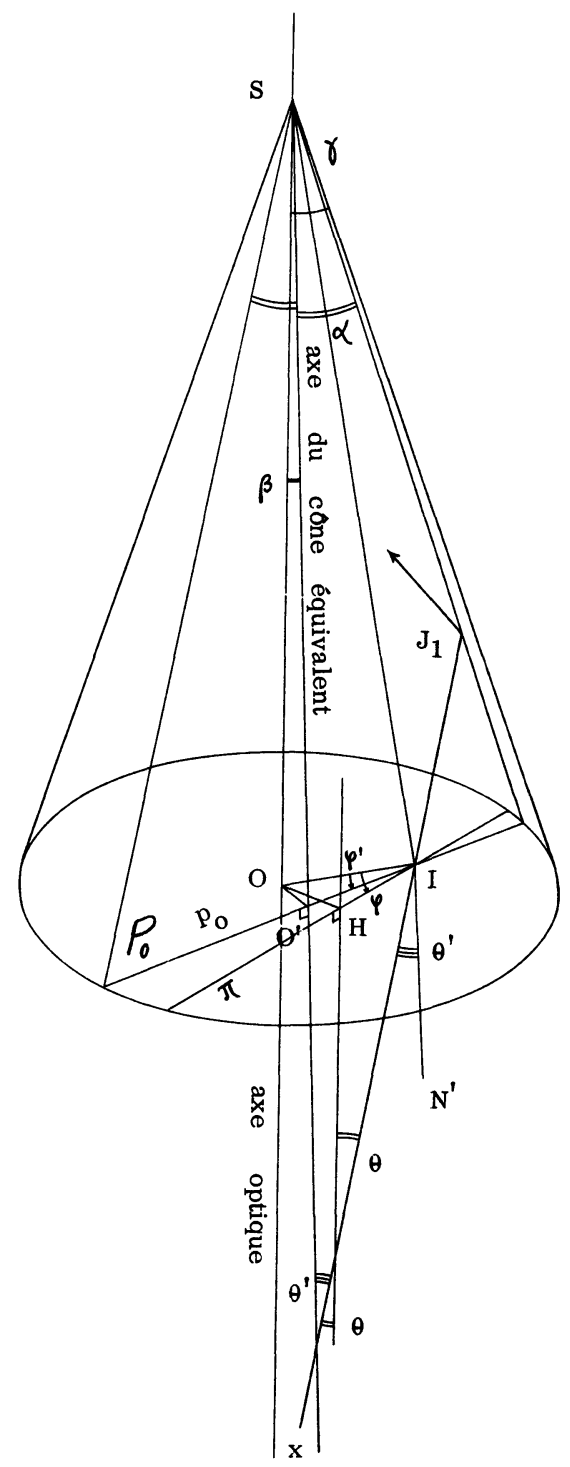

Fig. 12. - Cône équivalent pour un rayon non méridien. [Similar cone for a non-meridian ray.]

au point $I$ de la face d'entrée peut être représenté par trois paramètres : $r, \theta, \varphi$, choisis pour une pondération simple du flux et définis comme suit, en regard de la figure 13. 

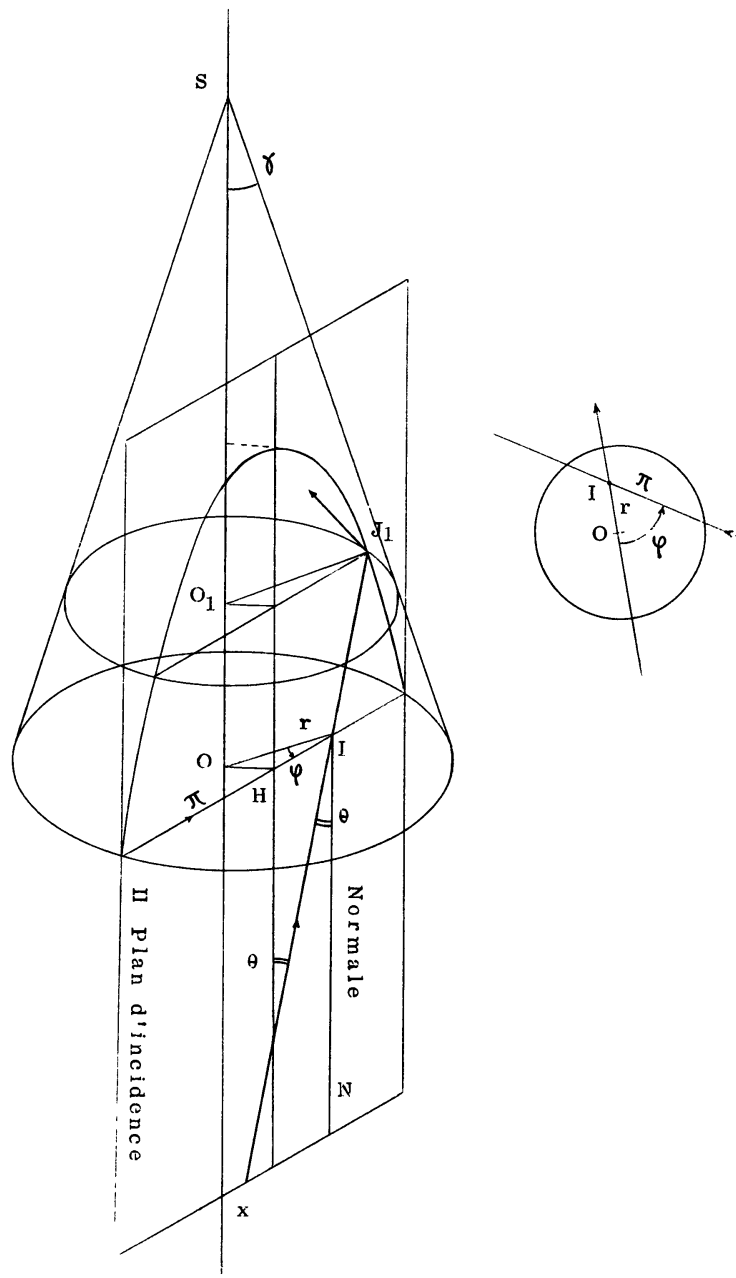

Fig. 13.

Soit $\Pi$ le plan d'incidence, $\pi$ la trace de $\Pi$ sur la face d'entrée, orientée comme projection du rayon $x I$. On pose :

$r=\mathrm{O} I \quad 0 \leqslant r \leqslant R \quad \begin{aligned} & O, \text { centre de la face d'entrée } \\ & R, \text { rayon de la face d'entrée }\end{aligned}$

$\theta$, angle d'incidence

$\varphi=(\mathbf{O I}, \pi) \quad 0 \leqslant \varphi \leqslant 2 \pi$.

Le choix de ces paramètres implique des hypothèses qui ne restreignent pas la généralité du problème. Essentiellement, on admet que le flux capté par la section d'entrée du cône, avec un demi-angle d'ouverture $\theta$, est de révolution autour de l'axe du miroir tronconique et est distribué selon la loi de Lambert. Avec ces hypothèses :
- la face d'entrée est éclairée uniformément. Le flux est donc pondéré en $r^{2}$, c'est-à-dire qu'une couronne comprise entre les cercles de rayons $r$ et $r+\mathrm{d} r$ reçoit un flux proportionnel à $2 \pi r \mathrm{~d} r$;

- le flux capté admet la symétrie de révolution. Il est donc pondéré en $\varphi$, c'est-à-dire que le flux transporté par des rayons incidents en un même point et de paramètre $\varphi$ compris entre $\varphi$ et $\varphi+\mathrm{d} \varphi$ est proportionnel à $\mathrm{d} \varphi$;

- conformément à l'énoncé du théorème, le flux capté est proportionnel à $\mathrm{d} \sin ^{2} \theta$.

On peut en conclure que l'élément de flux $d \Phi$, arrivant dans la couronne comprise entre les cercles de rayons $r$ et $r+\mathrm{d} r$ et, pour chaque point de cette couronne, dans la direction définie par $\varphi$ et $\theta$, à $\mathrm{d} \varphi$ et $\mathrm{d} \theta$ près est donné par :

$\mathrm{d} \Phi=K \mathrm{~d} r^{2} \mathrm{~d} \sin ^{2} \mathrm{~d} \varphi$

où $K$ est une constante de normalisation fixée selon le flux total $\Phi_{0}$ entrant dans le miroir conique par :

$\Phi_{0}=K 2 \pi R^{2} \sin ^{2} \theta$.

On remarquera que les conditions demandées sont satisfaites dans le cas usuel d'une source homogène, de petit angle apparent, située à l'infini, dont un flux de rayonnement est préconcentré au moyen d'une optique convergente aplanétique, de révolution autour de l'axe du miroir conique, donnant de la source une image qui coïncide avec la face d'entrée du cône. On vérifierait aisément que les conditions ci-dessus précisées pour la pondération du flux qu'on a adoptée, sont alors bien réunies.

L'expérience a montré qu'avec une optique frontale de mauvaise qualité (par exemple, lorsqu'on emploie des condenseurs ordinaires), les conclusions de la théorie conservent une signification utile.

Lorsque la distribution à l'entrée ne correspondra pas du tout à celle de ces hypothèses, il conviendra de reprendre la marche des calculs en tenant compte de la loi de distribution en cause, ou même, s'il n'y en a pas, en établissant un programme sur ordinateur qui pondère pinceau par pinceau élémentaire. Mais il faut souligner qu'on n'obtiendra jamais ainsi une aussi bonne optimisation qu'avec une distribution lambertienne. Aussi aura-t-on intérêt chaque fois que cela ne sera pas impossible, à conformer les éléments optiques pré-concentrateurs et à concevoir leur implantation, en vue de réaliser, au moins grosso modo, les conditions optimales qui sont prises ici comme base de départ.

3.1.2 Conclusions du calcul. - On trouve finalement pour le flux $\Phi_{(k)}$ parvenant en $k$ (en situant la section $k$ par rapport à la section $k_{2}$ de première optimisation, $k_{3}$ de seconde optimisation et $k_{5}$, telle que $k_{5}^{2}=k_{2} \cdot k_{3}$ ), lorsque $k_{2}<k<k_{5}:(12)$ 


$$
\begin{aligned}
\Phi(k) & =\pi R^{2} \sin ^{2} \theta_{1}+2 R^{2} \sin ^{2} \theta_{1}\left(\operatorname{Arc} \sin \frac{\sin \alpha_{2}}{\sin \gamma}-\frac{\sin \alpha_{2}}{\sin \gamma} \sqrt{1-\frac{\sin ^{2} \alpha_{2}}{\sin ^{2} \gamma}}\right)+ \\
& +\int_{\alpha 2}^{\gamma}\left\{\frac{4 R^{2} \sin ^{2} \alpha}{\sin \beta \sin ^{2} \gamma} \sin ^{2} \theta_{2}+\frac{2 R^{2}}{\sin \beta \sin ^{2} \gamma}(2 \sin \alpha \cos \alpha-\operatorname{tg} \alpha)\right. \\
& \times\left[\cos \theta_{1} \sqrt{\cos ^{2} \beta-\cos ^{2} \theta_{1}}-\cos \theta_{2} \sqrt{\cos ^{2} \beta \cos ^{2} \theta_{2}}\right] \\
& \left.-\frac{2 R^{2} \operatorname{tg} \alpha}{\operatorname{tg}^{2} \gamma \sin \beta}\left(\frac{1}{\cos ^{2} \alpha}-\frac{2}{k^{2}}\right)\left[\operatorname{Arc} \cos \frac{\cos \theta_{1}}{\cos \beta}-\operatorname{Arc} \cos \frac{\cos \theta_{2}}{\cos \beta}\right]\right\} \mathrm{d} \alpha
\end{aligned}
$$

et lorsque $k_{5}<k<k_{3}:$ (13)

$$
\begin{aligned}
\Phi(k) & =\pi R^{2} \sin ^{2} \theta_{1}-2 R^{2} \sin ^{2} \theta_{1}\left(\operatorname{Arc} \sin \frac{\sin \alpha_{3}}{\sin \gamma}-\frac{\sin \alpha_{3}}{\sin \gamma} \sqrt{1-\frac{\sin ^{2} \alpha_{3}}{\sin ^{2} \gamma}}\right)+ \\
& +\frac{4 R^{2}}{\sin \gamma \cos \alpha_{3}}\left(\alpha_{3}-\sin \alpha_{3} \cos \alpha_{3}\right) \sqrt{1-\frac{\sin ^{2} \alpha_{3}}{\sin ^{2} \gamma}-\frac{8 R^{2}}{k^{2}} \frac{\alpha^{3}}{\operatorname{tg}^{2} \gamma} \operatorname{Arg} \cosh \frac{\cos \alpha_{3}}{\cos \gamma}} \\
& +\frac{8 R^{2}}{k^{2} \operatorname{tg}^{2} \gamma} \int_{0}^{\alpha_{3}} \operatorname{Arg} \cosh \frac{\cos \alpha}{\cos \gamma} \mathrm{d} \alpha+\int_{\alpha_{3}}^{\gamma}\left\{\frac{4 R^{2} \sin ^{2} \alpha}{\sin \beta \sin ^{2} \gamma} \sin ^{2} \theta_{2}\right. \\
& +\frac{2 R^{2}}{\sin \beta \sin ^{2} \gamma}(2 \sin \alpha \cos \alpha-\operatorname{tg} \alpha)\left[\cos \theta_{1} \sqrt{\cos ^{2} \beta-\cos ^{2} \theta_{1}}-\cos \theta_{2} \sqrt{\cos ^{2} \beta-\cos ^{2} \theta_{2}}\right] \\
& \left.-\frac{2 R^{2} \operatorname{tg} \alpha}{\operatorname{tg}^{2} \gamma \sin \beta}\left(\frac{1}{\cos ^{2} \alpha}-\frac{2}{k^{2}}\right)\left[\operatorname{Arc} \cos \frac{\cos \theta_{1}}{\cos \beta}-\operatorname{Arc} \cos \frac{\cos \theta_{2}}{\cos \beta}\right]\right\} \mathrm{d} \alpha .
\end{aligned}
$$

Les bornes d'intégration $\alpha_{2}$ et $\alpha_{3}$ étant données par :

$\sin \alpha_{2}=\frac{k_{2} k_{3}-k_{2}}{k\left(k_{3}-k_{2}\right)} \sin \gamma \quad$ et $\quad \sin \alpha_{3}=\frac{k_{2}-k_{2} k_{3}}{k\left(k_{3}-k_{2}\right)} \sin \gamma$.

3.3 EVAluation DES PERTES PAR RÉFLEXIONS INTERNES. - On établit assez facilement que le nombre $p$ de réflexions subies par le plus défavorisé (celui qui va le moins loin), des rayons méridiens entrant sous l'incidence $\theta$ dans un miroir conique de demi-angle au sommet $\gamma$ est donné (en partie entière) par :

$p=\left[\frac{\frac{\pi}{2}-\theta}{2 \gamma}\right]$.

Pour évaluer les pertes de flux, il faut définir un nombre moyen, pondéré, de réflexion, $\bar{p}$, représentant la moyenne des nombres de réflexions subies par tous les rayons parvenant dans une section $k$, compte tenu du flux qu'ils transportent.

Pour obtenir ce nombre $\bar{p}$ on considère les rayons (méridiens ou non) d'incidence $\theta$ à l'entrée, progressant dans les cônes équivalents de demi-angle $\alpha$ (Voir Fig. 14).

Par rapport au nombre $p$ de réflexions du rayon méridien le plus défavorisé, on trouve comme valeur de majoration pour $\bar{p}$ :

$\bar{p}=\frac{2}{\pi} p \quad($ soit $=0,64 p)$.

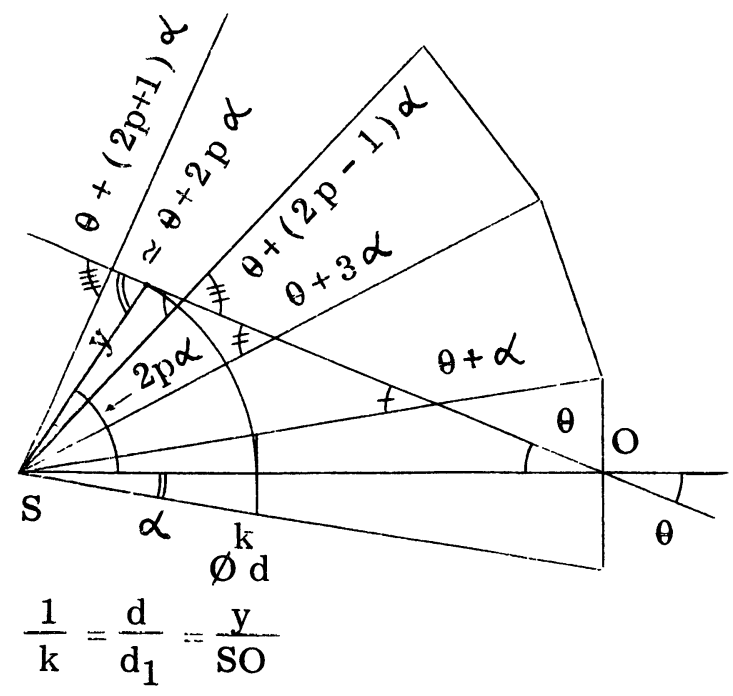

Fig. 14. - Réflexions subies par un rayon non méridien dans son cône équivalent.

[Reflections undergone by a non-meridian ray in its similar cone.]

Evaluons maintenant la perte de flux due aux seules réflexions internes dans un miroir conique. Désignons par $R$ le facteur de réflexion, par $\Phi_{1}$, le flux ayant 
pénétré dans le miroir par la face d'entrée $k_{1}$, par $\Phi$ le flux qui arrive dans la section $k$ après toutes les réflexions (abstraction faite de toute autre cause de perte de flux, notamment par absorption). Lorsque $R$ est assez proche de 1 et $\bar{p}$ pas trop grand, on peut écrire :

$\frac{\Phi}{\Phi_{1}}=R^{\bar{p}}$.

On peut préciser le domaine d'application de cette formule. Théoriquement, le rapport $\Phi / \Phi_{1}$ est donné par la moyenne de $R^{p}$. Or, on a toujours :

$R^{p}=\mathrm{e}^{p \log R}>1+\log R$

d'où :

$\frac{\Phi}{\Phi_{1}}=\overline{R^{p}}<1+\bar{p} \log R$.

Ceci nous donne une majoration de la perte de flux $\Delta \Phi$ :

$\frac{\Delta \Phi}{\Phi_{1}}<\bar{p}|\log R| \sim \bar{p}(1-R)$.

On peut donc appliquer à coup sûr la formule (16) toutes les fois qu'on peut assimiler, grosso modo, $\bar{p}(1-R)$ à $1-R^{\bar{p}}$.

Pour une évaluation très précise, ou bien dans le cas de distributions angulaires particulières à l'entrée, on établira un programme sur ordinateur en reprenant le calcul indiqué ci-dessus, au besoin pinceau par pinceau élémentaire.

4. Informations technologiques. - L'obtention des résultats qu'on peut attendre du recours à des miroirs coniques optimisés, dépend du coefficient de réflexion du matériau choisi pour ces miroirs. Le nombre moyen $\bar{p}$ de réflexions serait, par exemple, de 1,3 avec l'optimisation proposée plus haut (2.3). Il est essentiel que le coefficient de réflexion soit peu affecté par l'échauffement du matériau et que sa tenue à l'air libre dans le temps soit satisfaisante. Ces questions sont de la plus haute importance pour les applications. Les informations sont dispersées et certaines sont peu connues.

Le matériau de base qui s'impose est l'aluminium poli traité. Le choix réside dans la qualité d'aluminium et dans la nature du traitement.

Les qualités d'aluminium sont notées selon la pureté de l'alliage : A 5 pour $99,50 \%$; A 7 pour $99,70 \%$; A 8 pour $99,80 \%$; A 9 pour $99,998 \%$. Cette notation, en usage, n'est pas très logique (l'alliage à 99,90 n'existe pas). Ce matériau de base est livré avec un bon poli, en feuilles de diverses épaisseurs, protégées par un film plastique. Il existe en placages : par exemple, en A 9 mince plaqué sur A 4. Le fournisseur français est Cégédur-Péchiney.

Les traitements par brillantage électrolytique et oxydation anodique se partagent en procédés acides et procédés basiques selon que les électrolytes sont acides ou alcalins.

Ce n'est pas ici le lieu d'exposer ces procédés, mais seulement de faire état des résultats expérimentaux bien établis afin de fixer le meilleur choix pour les applications envisagées dans cet article.

Les principaux documents utilisés ont été : Les notes techniques publiées par l'Aluminium Français [18], Le Mémoire de M. Dupuy [19], La communication de N. D. Pullen [20], Les documents du Laboratoire d'Héliotechnique de la Faculté des Sciences de Marseille [21], Les documents du Laboratoire de Physique moléculaire de la Faculté des Sciences de Montpellier [22], Les mesures du Centre Technique de l'Aluminium [23], L'ouvrage de M. Schenk et A. Franke [24], L'article de J. H. Weaver [25]... A quoi se joignent des mesures faites depuis une dizaine d'années par l'auteur.

On verra qu'il ressort à l'évidence de ces informations que la meilleure formule est l'aluminium A 9, poli, traité par le procédé basique dit brytal. Le coefficient de réflexion se situe entre 0,87 et 0,94 . Il diminue de $3 \%$ environ au bout de 2 ans à l'air libre, de $5 \%$ au bout de 10 ans. Il demeure pratiquement sans diminution jusqu'à $500^{\circ} \mathrm{C}$ et est encore de 0,80 à $580^{\circ} \mathrm{C}$. Ces résultats apparaissent dans les figures et les tableaux suivants, empruntés aux documents consultés.

Ce procédé basique, connu sous la dénomination de "brytal » (marque déposée anglaise), est exploité en France par les Etablissements AMEL [26] et par la Société PROÉCLAIR [27]. Le procédé acide est plus économique et à préconiser pour d'autres types d'utilisation; mais le coefficient de réflexion obtenu est inférieur d'au moins $10 \%$. Aussi, le procédé basique est-il le seul dont les résultats soient analysés dans le présent article.

\subsection{COEFFICIENT DE RÉFLEXION APRÈS TRAITEMENT} RÉCENT ET A TEMPÉRATURE AMBIANTE. — La figure 15

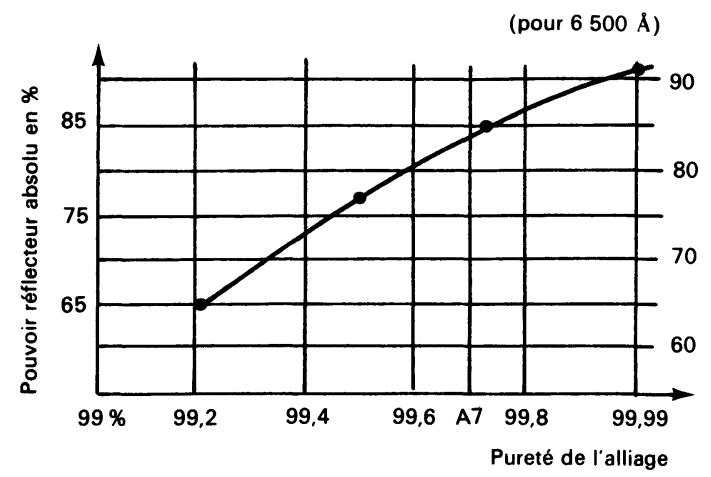

Fig. 15. - Variation de pouvoir réflecteur d'une surface d'aluminium brillantée par le procédé Brytal et renforcée par oxydation anodique en fonction de la pureté du métal. [18] Note technique de l'Aluminium Français (1953).

[Variation of the reflecting power of an Aluminium brillant surface by the method brytal and reinforced by anodic oxydation in function of metal purity.] 
montre les variations du coefficient de réflexion $R$ selon les qualités d'aluminium poli utilisé [18]; la figure 16, en fonction de la longueur d'onde [19]. On constate un fléchissement pour $0,800 \mu$. La figure 17 montre les variations de la même fonction pour un échantillon ayant subi un traitement spécial, dénommé Amel anti-infra [22] et [24]. Il n'y a plus de fléchissement et la courbe convient particulièrement bien pour le rayonnement solaire.

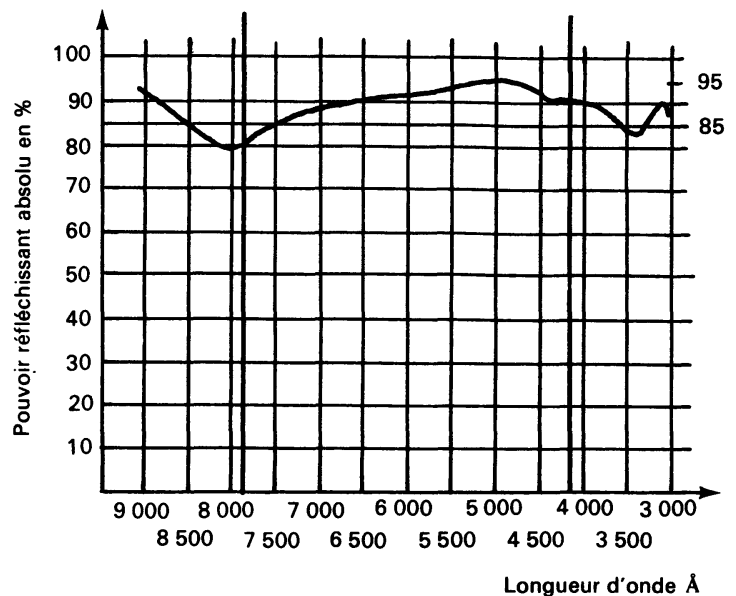

Fig. 16. - Variation du pouvoir réfléchissant de l'aluminium 99,9, traité par le procédé Brytal, en fonction de la longueur d'onde. Sans traitement anti-infra. [19] Mémoire de M. Dupuy, Ecole de Physique et Chimie (1942).

[Variation of the reflecting power of the 99 Aluminium treated with the brytal method in function of its wavelength, without anti-infra treatment.]

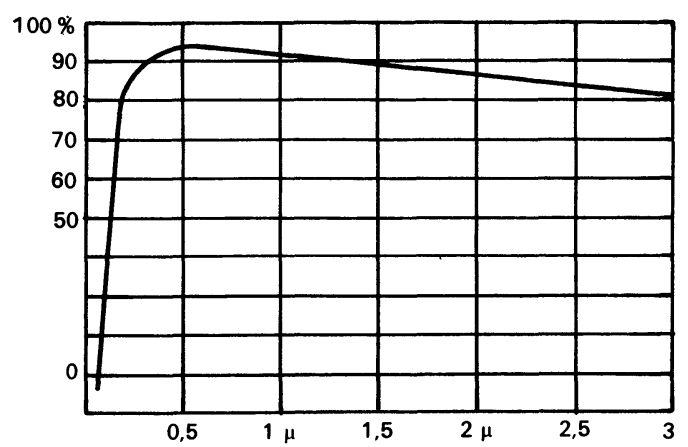

Fig. 17. - Après traitement anti-infra. [22] Labo physique moléculaire de la Faculté des sciences de Montpellier (1965).

[After anti-infra treatment.]

L'épaisseur habituelle de la couche d'alumine pure est $2 \mu$ (quoique le traitement opère davantage en profondeur), pour un coefficient $R$ de 0,89 . Les plus hautes valeurs $(0,94)$ sont obtenues en réduisant la durée du bain d'anodisation et l'épaisseur s'abaisse à $0,1 \mu$ [23]. La tenue dans le temps et en température est la même. Simplement, le nettoyage des pièces doit être effectué sans brutalité, au moyen d'un chiffon mouillé.

Quand on parle de coefficient de réflexion, on confond globalement, le plus souvent, la réflexion strictement spéculaire et la réflexion légèrement diffuse qui l'accompagne. Les proportions respectives de ces deux sortes de réflexion sont les suivantes; le coefficient global étant pris comme unité :

\begin{tabular}{lll} 
& $\begin{array}{c}\text { Réflexion } \\
\text { spéculaire }\end{array}$ & $\begin{array}{c}\text { Réflexion } \\
\text { diffuse }\end{array}$ \\
A 5 poli/brytal & $-\overline{\%}$ & - \\
A 8 poli/brytal & $93 \%$ & $7 \%$ \\
A 9 poli/brytal & $96,25 \%$ & $3,75 \%$ \\
Laiton plaqué argent & $99,4 \%$ & $0,6 \%$ \\
Laiton plaqué rhodium & $99,5 \%$ & $3,5 \%$ \\
Laiton plaqué chrome & $99,7 \%$ & $0,45 \%$ \\
\hline
\end{tabular}

4.2 Tenue DANS LE TEMPS, A L'AIR LIBRE. - La figure 18 montre les variations de $R$ sur 8 mois et demi, à l'air libre, pour divers matériaux réfléchissants [20].

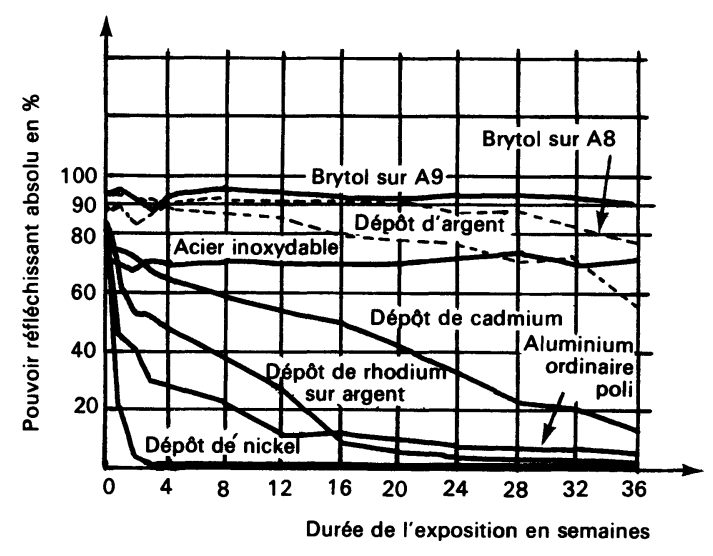

Fig. 18. - Variations avec le temps du pouvoir réfléchissant d'échantillons métalliques exposés à l'air libre. [20] Article N. D. Pullen (1936).

[Variation in time of the reflecting power of metalics samples at the open-air.]

Le tableau ci-après, portant sur un an et demi, a été publié dans les Notes Techniques de l'Aluminium Français [18] :

La tenue dans le temps observée en atmosphère marine est pratiquement la même [18]. Des mesures de l'auteur ont porté sur un intervalle de 10 ans. Il s'agit de pyramides réalisées en 1968 par PROÉCLAIR, en A 9 traité brytal, qui avaient à l'origine un coefficient de réflexion de 0,90 . Ces pyramides ont subi de nombreux échauffements et sont demeurées à l'air. Après nettoyage léger avec un chiffon humide, le coefficient mesuré en 1978 est de 0,85 .

Les mesures ont été effectuées par l'auteur au moyen d'un procédé simple. On place une cellule à couche 
Pouvoir réflecteur de différentes surfaces métalliques en fonction de leur durée d'exposition à l'air libre (pouvoir réflecteur en $\%$ du flux total de la lumière incidente).

\begin{tabular}{lrrrrrr}
\multicolumn{1}{c}{ Métal exposé } & \multicolumn{1}{c}{1} & 1 mois & 3 mois & 6 mois & 1 an & 1 an $1 / 2$ \\
Acier inoxydable & - & - & - & - & - & - \\
Dépôt d'argent poli & $68 \%$ & $68 \%$ & $68 \%$ & $68 \%$ & $67 \%$ & $67 \%$ \\
Dépôt de rhodium s/argent & $98 \%$ & $88 \%$ & $83 \%$ & $70 \%$ & $29 \%$ & $5 \%$ \\
Dépôt de nickel s/laiton & $85 \%$ & $48 \%$ & $24 \%$ & $5 \%$ & $2 \%$ & $2 \%$ \\
Dépôt de cadmium s/laiton & $78 \%$ & $3 \%$ & $2 \%$ & $2 \%$ & $2 \%$ & $2 \%$ \\
Dépôt de chrome s/laiton & $74 \%$ & $65 \%$ & $55 \%$ & $32 \%$ & $13 \%$ & $2 \%$ \\
Aluminium ordinaire poli & $63 \%$ & $46 \%$ & $8 \%$ & $7 \%$ & $3 \%$ & $3 \%$ \\
Aluminium $99,99 \%$ traitén par le procédé basique, & $73 \%$ & $31 \%$ & $11 \%$ & $3 \%$ & $2 \%$ & $2 \%$ \\
avec protection & $90 \%$ & $89 \%$ & $87 \%$ & $86 \%$ & $86 \%$ & $86 \%$
\end{tabular}

d'arrêt, au sélénium, à une distance donnée d'un petit laser (CSF, de 0,2 $\mathrm{mW}$, rouge, en l'occurrence). On note un chiffre sur le galvanomètre. Puis, à la moitié de cette distance, on dispose légèrement en biais l'échantillon réfléchissant à mesurer et, dans le plan de sortie du laser, on recueille sur la cellule le rayon réfléchi. On note un second chiffre. Le coefficient de réflexion spéculaire est égal au rapport du second chiffre au premier. Si l'on désire mesurer le coefficient global (réflexion spéculaire + réflexion diffuse autour du rayon réfléchi), il suffit d'avoir une cellule assez grande et de la disposer assez près du miroir pour recueillir le flux dans un grand angle.

4.3 TENUE EN TEMPÉRATURE. - La figure 19 montre les variations de $R$ en fonction de la température pour divers matériaux réfléchissants [20].

Les Notes Techniques de l'Aluminium Français [18], donnent le tableau suivant :

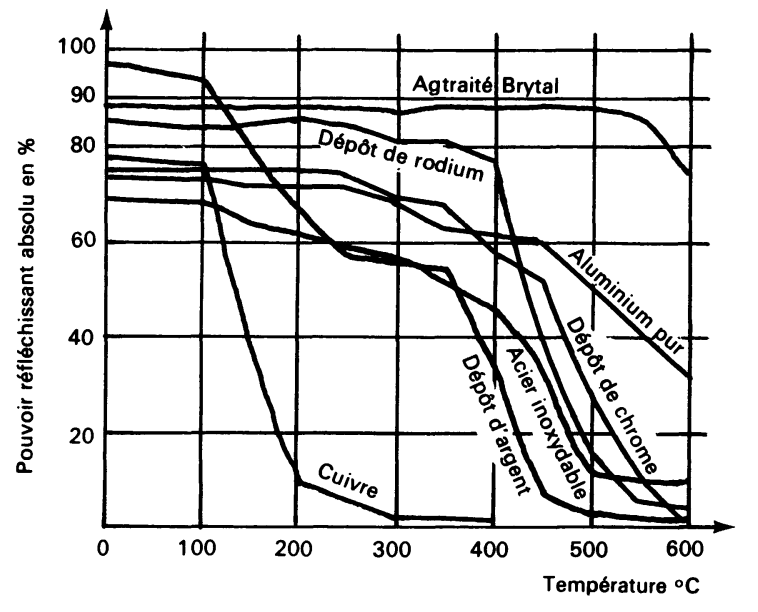

Fig. 19. - Influence de la température sur le pouvoir réfléchissant des surfaces métalliques. [20] N. D. Pullen (1936).

[Influence of the temperature on the reflecting power of the metalic surface.]

Pouvoir réflecteur en fonction de la température d'utilisation.

\begin{tabular}{|c|c|c|c|c|c|c|c|}
\hline & & & Ter & érature & ${ }^{\circ} \mathrm{C}$ & & \\
\hline Métal exposé & $0^{\circ}$ & $100^{\circ}$ & $200^{\circ}$ & $300^{\circ}$ & $400^{\circ}$ & $500^{\circ}$ & $600^{\circ}$ \\
\hline - & - & - & - & - & - & - & - \\
\hline Acier inoxydable poli & $68 \%$ & $61 \%$ & $58 \%$ & $47 \%$ & $10 \%$ & $10 \%$ & $9 \%$ \\
\hline Argent poli & $98 \%$ & $95 \%$ & $64 \%$ & $55 \%$ & $33 \%$ & $4 \%$ & $3 \%$ \\
\hline Rhodium poli & $85 \%$ & $84 \%$ & $84 \%$ & $81 \%$ & $78 \%$ & $18 \%$ & $5 \%$ \\
\hline Chrome poli & $63 \%$ & $63 \%$ & $62 \%$ & $60 \%$ & $49 \%$ & $24 \%$ & $2 \%$ \\
\hline Aluminium ordinaire $(99,5)$ poli & $73 \%$ & $73 \%$ & $72 \%$ & $66 \%$ & $59 \%$ & $48 \%$ & $33 \%$ \\
\hline $\begin{array}{l}\text { Aluminium à } 99,99 \% \text { traité par le procédé } \\
\text { basique, protégé }\end{array}$ & $90 \%$ & $90 \%$ & $90 \%$ & $90 \%$ & $90 \%$ & $89 \%$ & $76 \%$ \\
\hline
\end{tabular}

5. Conclusion. - Cette étude a exposé les données prospectives d'une nouvelle optimisation qu'apporterait aux projets de centrales solaires à tour et champ d'héliostats, l'introduction d'un miroir tronconique adéquat, en terminal de concentration optique. L'état de la technologie des miroirs en aluminium poli et traité en rendrait, semble-t-il, la réalisation parfaitement possible. 


\section{Bibliographie}

[1] Malifaud, P., La concentration optique maximale dans les récepteurs de rayonnements. Les miroirs coniques optimisés. Thèse, Paris, 1968 (400p.).

[2] Malifaud, P., Thermodynamique et optique géométrique. L'entropie optique. Table ronde du C.N.R.S. Sadi Carnot et l'essor de la thermodynamique (1974) (Editions du C.N.R.S.) 1976.

[3] Malifaud, P., Traitement thermodynamique des problèmes d'optique géométrique touchant la concentration et la projection des flux de rayonnement. Rev. Gén. Electr. 82 (1973).

[4] Malifaud, P., Nouvelles techniques d'éclairage par miroirs coniques optimisés. $4^{\mathrm{e}}$ Colloque International de Cinématographie rapide. Collection de l'A.N.R.T., 1975.

[5] Malifaud, P., Brevet français no 1.543 .165 du 6/5/64 (163 p.).

[6] Malifaud, P., Brevet français $\mathrm{n}^{\circ} 1.587 .607 \mathrm{du} 12 / 6 / 68$.

[7] Malifaud, P., Brevet français $n^{\circ} 1.576 .599 \mathrm{du} 12 / 6 / 68$.

[8] Malifaud, P., Brevet français $\mathrm{n}^{0} 1.576 .600 \mathrm{du} 12 / 6 / 68$.

[9] Malifaud, P., Brevet français $\mathrm{n}^{\circ} 1.576 .601 \mathrm{du} 12 / 6 / 68$.

[10] Malifaud, P., Brevet français $\mathrm{n}^{\circ} 1.602 .203 \mathrm{du}$ 19/7/68.

[11] Malifaud, P., Brevet français no 2.059.454 du 7/9/69.

[12] Malifaud, P., Brevet français $\mathrm{n}^{0} 2.070 .286$ du 3/4/73.

[13] Malifaud, P., Brevet français $n^{0} 2.070 .287$ du 3/4/73.

[14] Malifaud, P., Brevet français $n^{0} 2.070 .288$ du 3/4/73.

[15] Malifaud, P., Augmentation de concentration du rayonnement solaire par réflexion au niveau du foyer, sur des surfaces de formes adéquates. Cahiers de l'A.F.E.D.E.S. no 3 (1971)
[16] Projet THEM $77(02,03)$. C.N.R.S./E.D.F. Rapports internes E.D.F., articles, notamment, de MM. Mersier, Bonduelle, Denayrolles, Gentili, Sicart.

[17] Wernick, S. et Pinner, R., Les traitements de surface et la finition de l'aluminium. Eyrolles (1962).

[18] Notes Techniques de l'Aluminium Français, 23, rue Balzac, Paris $8 \mathrm{e}$.

[19] Dupuy, M., Sous-Chef de Travaux à l'Ecole de Physique et Chimie. Mémoire du 20 juin 1942.

[20] Pullen, N. D., J. Inst. Met. no 2 (1936).

[21] Laboratoire d'Héliotechnique de la Faculté des Sciences de Marseille Saint-Jérôme. Communication et documents techniques (1970).

[22] Laboratoire de Physique moléculaire de la Faculté des Sciences de Montpellier. Professeur Mlle Vergnoux (1965).

[23] Centre technique de l'Aluminium, 86, boulevard de Grenelle, Paris $15 \mathrm{e}$.

[24] Schenk, M. et Francke, A., Werkstoff Aluminium und Seine anodische oxydation (A. G. Verlag, Bern).

[25] Weaver, J. H., Bright Anodized Temperature Control Coatings Effect on Optical Properties. American Electroplaters Society, San Francisco, July 1968, Plating.

[26] Etablissements AMEL, 26 bis, rue Eugène-Eichenberger; Puteaux. Documents et échantillons communiqués par M. Gasser, Directeur.

[27] Société PROÉCLAIR, 11, rue de l'Hôpital-Saint-Louis, Paris $10^{\mathrm{e}}$. 